\title{
Histoire de la tomate à travers son génome
}

> La tomate, pomme d'amour ou pomme d'or, Simone Gilgenkrantz dont le nom nous vient des Aztèques (Xictomatl en Natualt) fut d'abord en France une plante ornementale, suspecte aux yeux des botanistes du fait de sa parenté avec la belladone dont ils connaissaient la toxicité. Mais son fruit fut cependant consommé en Espagne dès le XVI ${ }^{e}$ siècle et il est devenu peu à peu partie intégrante de toutes les cuisines du monde. Consommée fraîche, en jus, en sauces, en concentrés, en condiments, la tomate fait aujourd'hui l'objet d'une production industrielle considérable ainsi que de nombreuses recherches scientifiques. Le décryptage de son génome, qui vient d'être réalisé, permettra de nouveaux progrès dans sa conservation, sa saveur, la création de nouvelles variétés. Il devrait être rapidement suivi par celui des génomes d'autres solanacées. «

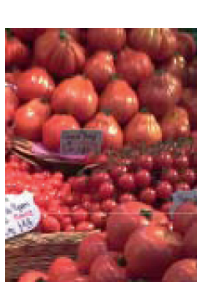

médecine/sciences,

9 , rue Basse,

54330 Clérey-sur-Brénon,

France.

simsimone.gilgenkrantz@

gmail.com

sauvages. Considérée comme un modèle en génétique, c'est la seule plante qui eut une variété génétiquement modifiée autorisée à la consommation humaine, mais durant quelques années seulement. D'abord ingrédient important de la gastronomie des cultures méditerranéennes, consommée crue, cuite, séchée, en conserve, jus, sauce ou concentré, elle s'est mondialisée ; on la trouve aussi bien au Moyen Orient qu'en Asie où elle garde toujours son nom. En raison de son importance

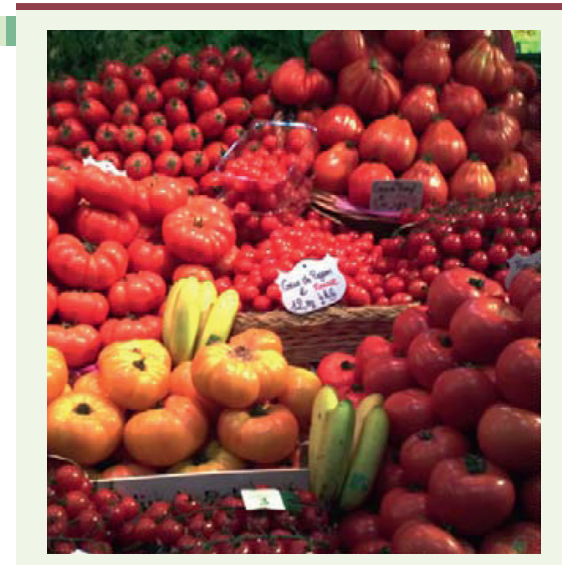

(c) Simone Gilgenkrantz

Le génome de la tomate domestique (Solanum lycopersicum) ${ }^{1}$ vient d'être séquencé par le Tomato Genome Consortium (TGC). Cette plante, d'origine péruvienne, est aujourd'hui mondialement cultivée (sur environ trois millions d'hectares). Son fruit, classiquement rond, charnu, et d'un rouge roboratif est entré depuis quelque temps dans sa période baroque. Désormais sur les marchés apparaissent des tomates « cœur de bœuf » ou « cœur de pigeon », en grappe, côtelées, oblongues, et de toutes couleurs, vert, jaune, violet ou noir. De nombreuses équipes, dont celles de l'Institut national de la recherche agronomique (Inra) en France, ont isolé des gènes de Solanum lycopersicum et des espèces

${ }^{1}$ Les Solanacées sont une famille de plantes appartenant à l'ordre des solanales au sein de laquelle on trouve la tomate, la pomme de terre, le poivron, l'aubergine. économique et de ses capacités d'hybridation, elle a fait l'objet de nombreuses recherches et, depuis le séquençage de son génome, elle possède désormais un site [1].

\section{Origine et domestication}

Les premières tomates proviennent des régions andines de la côte nord-ouest d'Amérique du Sud où diverses espèces, dont Solanum lycopersicum cerasiforme (la tomate cerise) et Solanum pimpinellifolium, se multiplient spontanément. Il semble que sa domestication ait eu lieu au Mexique : les tomates à gros fruits existaient déjà lors de la conquête du pays par les Espagnols en 1519. Ceuxci l'introduisirent rapidement en Europe, mais d'abord comme ornement pour jardins et tonnelles - les plants étaient alors arborescents - car du fait de son apparentement à la belladone (Atropa belladonna), bien connue pour sa toxicité, elle fut jugée non comestible. Puis elle fut ajoutée en Espagne au gaspacho (soupe 
andalouse de légumes crus consommée froide). Elle conquiert ensuite I'Italie. Ce n'est que vers la fin du XvIII ${ }^{e}$ siècle qu'elle entra dans la cuisine française (le catalogue Vilmorin-Andrieux la classe encore comme plante ornementale en 1760).

\section{Hybridations}

Au $x I x^{e}$ siècle, les sociétés d'agriculture se multiplient. Les techniques d'hybridation sont appliquées à l'amélioration des espèces agricoles, sans que soient encore comprises les lois qui les régissent (les travaux de Mendel ne seront publiés que bien plus tard en 1866). En 1856, les premières variétés (au nombre de sept) marquent le début des recherches et l'obtention des hybrides [3]. Celles-ci ont une faible variété génétique du fait du goulot d'étranglement que représente le transfert de la tomate domestique en Europe. Charles M. Rick fut un des premiers à organiser des expéditions dans la cordillère des Andes pour rechercher des variétés sauvages afin d'effectuer des croisements. C'est ainsi que Lycopersicon esculentum var. cerasiforme ou tomate cerise, solanum pimpinellifolium ou tomate groseille pour n'en citer que quelques-unes - ont permis d'obtenir des lignées nouvelles par introgression ${ }^{2}$. Le Rick tomato genetics resource center dispose à présent de plus de 1500 variétés. Une tomate bleue (OSU P20 pour Oregon state university $20^{\mathrm{e}}$ d'une série d'essais) a même été obtenue par croisement entre une tomate domestique et une tomate sauvage du Pérou, riche en anthocyanine. Aujourd'hui, la tomate domestique comporte des milliers de cultivars.

\section{Les sauces tomate et l'industrialisation}

En Europe, sauces, coulis, concentrés sont préparés avec diverses plantes aromatiques selon les pays. Du fait de sa fragilité, la transformation industrielle de la tomate est importante. Aux États-Unis, elle a connu une destinée particulière, grâce à la fabrication d'une sauce à base de tomates plutôt sucrées de l'espèce Solanum pennelli (la cause moléculaire des variations de glucose et de sucrose selon les espèces a été expliquée récemment [2]). Après quelques tentatives incertaines, Henry J. Heinz réussit à commercialiser le Tomato Ketchup en 1872 . Ce condiment à la saveur aigre douce supplanta bientôt tous les autres aux États-Unis. Après la Seconde Guerre mondiale, le ketchup accompagne la «macdonalisation » de l'alimentation en Europe, puis dans les autres continents : grâce à un marketing imparable, la fastfood risque de supplanter peu à peu les cuisines traditionnelles.

\section{Étude moléculaire et tomates transgéniques}

Vers 1990, la société Calgene met au point une tomate transgénique moins fragile (moins sensible au pourrissement). Elle peut être récoltée à maturité complète alors que les tomates non modifiées sont récoltées avant leur mûrissement qui est obtenu grâce à l'éthylène, jouant le rôle d'une hormone végétale. La production de polygalac-

\footnotetext{
${ }^{2}$ Introgression : dispersion des gènes d'une espèce à l'intérieur du pool génétique d'une autre espèce.
}

turonase, responsable de la fragilité par dégradation des parois cellulaires, peut être freinée par introduction d'un ARN antisens diminuant l'expression du gène correspondant. Cette tomate, Flavr Savr, obtient de la Food and drug administration (FDA) une autorisation de mise sur le marché en 1994 (sans étiquetage particulier). Pourtant, l'ajout d'un marqueur génétique (une résistance à la kanamycine) aurait pu poser problème. En fait, les tomates Flavr Savr n'eurent pas de succès auprès des consommateurs et elles furent retirées du marché en 1996.

Au Royaume-Uni, la société Zeneca produit un concentré de tomate plus épais, à base de tomate transgénique à pulpe plus dense. Bien qu'étiqueté organisme génétiquement modifié (OGM), il eut un grand succès jusqu'à la crise de la vache folle qui rendit les consommateurs plus précautionneux.

\section{Le séquençage de la tomate}

Les études moléculaires déjà effectuées par de nombreuses équipes méritaient d'être coordonnées et complétées. En 2003, à Washington, le Consortium du génome de la tomate (TGC) est créé et prend la décision de séquencer entièrement le génome de Solanum lycopersicum. Cette tâche considérable, qui a duré plusieurs années, vient d'être menée à son terme [4]. Quatorze pays regroupant plus de 300 chercheurs y ont participé. En France, les chercheurs de l'Inra qui avaient été à l'origine du projet ont joué une part importante dans ce travail, coordonné par le Centre Inra Toulouse Midi-Pyrénées.

La variété choisie fut «Heinz 1706 », développée en 1960 et originaire des États-Unis [5]. La tomate domestique possédant 12 chromosomes, chacun d'eux a été attribué à un pays, la France étant chargée du chromosome 7. L'analyse des chromosomes a montré que la région péricentrique est surtout hétérochromatique, alors que les extrémités distales sont euchromatiques. Sur les $900 \mathrm{Mb}, 760 \mathrm{Mb}$ ont été assemblés et alignés sur les 12 chromosomes, avec cartographie par FISH (hybridation in situ en fluorescence). La séquence complète a été obtenue par étapes successives: recherche des régions riches en gènes, puis séquençage à haut débit par les séquenceurs de nouvelle génération (NSG). L'ensemble des différentes opérations, leur chronologie, ainsi que les équipes impliquées est représenté dans la figure 33 du supplément $n^{\circ} 1$ de [4].

La comparaison entre le génomes de $S$. lycopersicum et un draft du génome de S. pimpinellifolium montre une divergence d'environ 0,6\%. L'ordre, l'orientation et la localisation des 35000 gènes ont été obtenus. Ceux-ci 
ont été étudiés par comparaison avec ceux de la pomme de terre, du riz, du raisin et d'Arabidopsis.

Un pipeline d'annotation entre les génomes de la tomate et de la pomme de terre, qui ont $8 \%$ de divergence, a permis de comparer les 18320 gènes orthologues. Les mutations non synonymes ${ }^{3}$ sont plus fréquentes que les modifications synonymes. Ceci peut indiquer une sélection diversifiante entre ces deux plantes, l'une privilégiant le fruit, et l'autre les tubercules.

Le goulot d'étranglement - du fait d'un passage réduit de graines de tomate domestique en Europe au $x{ }^{e}$ siècle - se reflète dans la comparaison des génomes de $S$. lycopersicum et de S. pimpinellifolium. Cependant, grâce aux hybridations interspécifiques, des gènes de 7/9 des espèces sauvages connues se retrouvent par introgression dans la tomate domestique.

Enfin, deux événements de polyploïdisation sont intervenus au cours de l'évolution. Il y a 60 millions d'années est survenue une triplication du génome. Il est intéressant de noter qu'elle coïncide avec la période d'extinction brutale des espèces sur terre. Elle pourrait expliquer le développement des Solanacées. La plupart de ces éléments dupliqués ont ensuite disparu mais, parmi ceux qui persistent, nombreux sont ceux qui portent des gènes contrôlant l'accumulation des pigments antioxydants au cours de la maturation des fruits.

\section{Perspectives}

Grâce à cette connaissance précise du génome de la tomate, modèle pour les plantes à fruits, de nouvelles publications apparaissent sur les progrès dans la maturation [6], la résistance au froid et la reproduction des Solanacées. Au-delà de la tomate, c'est toute la famille des Solanacées, première source alimentaire après les céréales, qui va tirer profit de cette avancée: poivron, piment, aubergine, mais aussi tabac ou encore pomme de terre. Du reste, ce travail n'est qu'un début puisqu'il s'inscrit dans un projet plus vaste : the international Solanaceae genome project. $\diamond$

${ }^{3}$ Les mutations non synonymes produisent des changements d'acides aminés à l'opposé des synonymes qui n'en induisent pas.

\section{SUMMARY}

Story of the tomato through its genome

Tomato (Solanum lycopersicum) is a model for fruit development. The tomato history has origins traced back to the early Aztecs. It was not until around the $16^{\text {th }}$ century that Europeans were introduced to this fruit, but only as ornemental plant since it was related to nightshade belladona. Then it was accepted into the kitchen all around the world. The genome sequence of the inbred cultivar Heinz 1706 is sequenced and provides interesting insights into the fleshy evolution. $\diamond$

\section{LIENS D'INTÉRÊT}

L'auteure déclare n'avoir aucun lien d'intérêt avec les données publiées dans cet article.

\section{RÉFÉRENCES}

\section{1. http://solgenomics.net}

2. Fridman $\varepsilon$, Pleban T, Zamir D. A recombination hotspot delimits a wildspecies quantitative trait locus for tomato sugar content to $484 \mathrm{bp}$ within an invertase gene. Proc Natl Acad Sci USA 2000 ; 97 : 4718-23.

3. Pitrat M, Cootd F. Histoire de légumes. La tomate. Paris : Inra, 2003.

4. The tomato genome consortium. The tomato genome sequence privides insights into fleshy fruit evolution. Nature $2012 ; 485: 635-41$.

5. Ozminkowsi R. Pedigree of variety Heintz 1706. Rep Tomato Genet Coop $2004 ; 54: 26$.

6. Powell ALT, Nguyen CV, Hill T, et al. Uniform ripening encodes a golden-2-like transcription factor regulating tomato fruit chloroplast development. Science 2012 ; 336 : 1711- 5 .

7. Mboup M, Fischer I, Lainer H, Stephan W. Trans-species polymorphism and allele-specific expression in the CBF gene family of wild tomatoes. Mol Biol Evol 2012 (sous presse).

8. Ruan YL, Patrick JW, Bouzayen M, et al. Molecular regulation of seed and fruit set. Trends Plant Sci 2012 (sous presse).
Tarifs d'abonnement $m / s-2013$

$>$ Grâce à $m / s$, vivez en direct les progrès des sciences biologiques et médicales

\section{Abonnez-vous}

à médecine/sciences
Bulletin d'abonnement page 1018 dans ce numéro de $\mathrm{m} / \mathrm{s}$

\section{TIRÉS À PART}

S. Gilgenkrantz

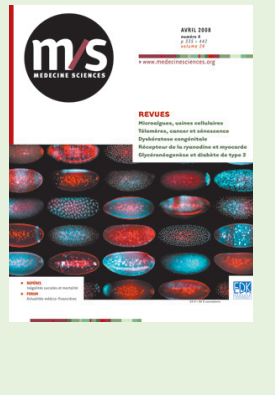

